The University of San Francisco

USF Scholarship: a digital repository @ Gleeson Library | Geschke Center

2010

\title{
The Effects of Opposition and Gender on Knee Kinematics and Ground Reaction Force During Landing From Volleyball Block Jumps
}

Gerwyn Hughes

University of San Francisco, ghughes@usfca.edu

James Watkins

Nick Owen

Follow this and additional works at: https://repository.usfca.edu/ess

Part of the Sports Sciences Commons, and the Sports Studies Commons

\section{Recommended Citation}

Hughes, Gerwyn; Watkins, James; and Owen, Nick, "The Effects of Opposition and Gender on Knee Kinematics and Ground Reaction Force During Landing From Volleyball Block Jumps" (2010). Kinesiology (Formerly Exercise and Sport Science). 34.

https://repository.usfca.edu/ess/34 
1 Title: The effects of opposition and gender on knee kinematics and ground reaction force during landing from volleyball block jumps.

4 Corresponding author: Dr. Gerwyn Hughes.

5 Postal Address: Division of Sport, Health and Exercise, University of Hertfordshire,

6 College Lane, Hatfield, AL10 9AB.

$7 \quad$ Email: $\quad$ g.hughes@ $@$ herts.ac.uk

$8 \quad$ Telephone: $\quad+441707289430$

9

10 Author information: Gerwyn Hughes is a lecturer in the Division of Sport Health and

11 Exercise at the University of Hertfordshire, UK. James Watkins is a Professor and Nick

12 Owen is a lecturer in the Department of Sports Science at Swansea University, UK. 
14 Title: The effects of opposition and gender on knee kinematics and ground reaction force during landing from volleyball block jumps. 


\section{Abstract}

The aim of the study was to examine the effect of opposition and gender on knee kinematics and ground reaction force during landing from a volleyball block jump. Six female and six male university volleyball players performed two landing tasks 1) an unopposed and 2) an opposed volleyball block jump and landing. Knee kinematics were recorded by a 12 camera motion analysis system $(120 \mathrm{~Hz})$ and ground reaction force was recorded by a force platform $(600 \mathrm{~Hz})$ during landing. The results showed a significant effect for level of opposition in peak normalized GRF $(p=.04)$, knee flexion at ground contact $(p=$ $.003)$, maximum knee flexion $(p=.001)$ and range of motion of knee flexion $(p=.003)$. There was a significant effect for gender in maximum knee flexion $(p=.01)$, range of motion of knee flexion $(p=.001)$, maximum knee valgus angle $(p=.001)$ and range of motion of knee valgus $(p=.001)$. The changes in landing biomechanics as a result of opposition suggest future research investigating landing mechanics should examine opposed exercises since opposition may significantly alter neuromuscular responses. 
The effects of opposition and gender on knee kinematics and ground reaction force during landing from volleyball block jumps.

Research suggests that approximately $70 \%$ of anterior cruciate ligament (ACL) injuries occur in sporting activities (Faegin, 1988; Johnson, 1988; Smith, Livesay, \& Woo, 1988). Studies examining the etiology of ACL injuries report that between $70 \%$ and $90 \%$ of injuries occur in non-contact situations (Griffin et al., 2000; McNair, Marshall, \& Matheston, 1993; Mykelbust, Maehlum, Engbretsen, Strand, \& Solheim, 1997). Furthermore, the incidence of ACL injuries is high in sports which involve a high frequency of landing (Hopper \& Elliot, 1993), decelerating (Miller, Cooper, \& Warner, 1995) or rapidly changing direction (Arendt \& Dick, 1995; Griffin et al., 2000; Olsen, Mykelbust, Engebretsen, \& Bahr, 2004), such as basketball, netball, handball and volleyball. The incidence of non-contact ACL injuries have been reported to be 6 to 8 times greater in females than in males competing in the same sports (Arendt \& Dick, 1995; Chandy \& Grana, 1985; Ferretti, Papandrea, Conteduca, \& Mariani, 1992; Gray et al., 1985; Gwinn, Wilckens, McDevitt, Ross, \& Kao, 2000; Lidenfeld, Schmitt, Hendy, Mangine, \& Noyes, 1994; Malone, Hardaker, Garrett, Feagin, \& Bassett, 1993).

Since ACL injuries have been associated with landing, decelerating and rapidly changing direction, a number of studies have investigated gender differences the biomechanics associated with these maneuvers (Decker, Torry, Wyland, Sterett, \& Steadman, 2003; Ford, Myer, \& Hewett, 2003; James, Sizer, Starch, Lockhart, \& Slauterbeck, 2004; Kernozek, Torry, Van Hoof, Cowley, \& Tanner, 2005; Malinzak, Colby, Kirkendall, Yu, \& Garrett, 2001; Yu, Lin, \& Garrett, 2006). Studies examining sagittal plane kinematics of landing and cutting maneuvers report that females tend to land with less knee flexion angle than males (Decker et al., 2003; James et al., 2004; Malinzak et al., 2001; Yu et al., 2006) and exhibit a greater range of knee flexion than males (Decker et al., 2003). Due to the effect of 
knee flexion on the patella tendon-tibia shaft angle, when a given load is acting through the patellar ligament there is likely to be a greater strain placed on the ACL if the knee flexion angle is small (Li et al., 1999; Nunley, Wright, Renner, Yu, \& Garrett, 2003). A number of observational studies including Boden et al. (2000) and Olsen et al. (2004) have reported that non-contact ACL injuries most frequently occur immediately following initial ground contact with the knee close to full extension. Consequently, since females tend to make contact with the ground with knees in a more extended position than males, the risk of ACL injury may be greater in females relative to males. Studies investigating frontal plane kinematics of landing and cutting report that females tend to exhibit greater maximum knee valgus angle and greater knee valgus angle range of motion compared to males (Ford et al., 2003; Kernozek et al., 2005; Malinzak et al., 2001). Boden et al. (2000) and Olsen et al. (2004) have reported that non-contact ACL injuries appear to occur more frequently when the knee exhibits a valgus movement. Consequently, greater maximum knee valgus angle in females may increase the risk of ACL injury relative to males. Some studies also suggest that females exhibit greater normalized peak ground reaction force (GRF) during landing than males (Kernozek et al., 2005; Salci, Kentel, Heycan, Akin, \& Korkusus, 2004; Yu et al., 2006). The greater the GRF exhibited during landing, the greater the likely load on the passive support structures of the knee and therefore the greater the likelihood of injury (Devita \& Skelly, 1992).

The demands of the tasks that participants are required to perform will influence the movement patterns exhibited and therefore influence the validity of comparisons made between males and females. Previous studies examining landing biomechanics in males and females typically use tasks involving a stop-jump (Chappell, Yu, Kirkendall, \& Garrett, 2002; Yu et al., 2006; Yu et al., 2005), a maximum height vertical jump (Hewett, Stroupe, Nance, \& Noyes, 1996; Swartz, Decoster, Russell, \& Croce, 2005) or dropping down from a raised 
platform set at the same height for both males and females (Decker et al., 2003; Ford et al., 2003; Kernozek et al., 2005; Salci et al., 2004). Dropping down from a raised platform may result in significantly different task demands for females compared to males (females are less likely to jump as high as females), particularly in sports such as volleyball where the net is set at a different height for males and females (2.48 $\mathrm{m}$ for males and $2.29 \mathrm{~m}$ for females). Therefore, a lack of standardization in the task participants are required to perform in previous studies may have reduced the likelihood of meaningful comparison between males and females. Previous studies have found changes in technique as a result of opposition (Davila, Garcia, Montilla, \& Ruiz, 2006). For example, Davila et al. (2006) found significant changes in technique were made by a handball players when shooting during unopposed and opposed conditions. It is reasonable to assume that the attentional demand of jumping and landing in an opposed context will be less than that in an unopposed context (Chen et al., 1996; Lajoie, Teasdale, Bard, \& Fleury, 1993) which, in turn, is likely to affect the neuromuscular response when landing. Despite this, the vast majority of studies examining gender differences in kinematics and kinetics during landing and cutting maneuvers use an unopposed task (Decker et al., 2003; Kernozek et al., 2005; Salci et al., 2004; Yu et al., 2006), with only a small number of studies examining opposed tasks (Hughes, Watkins, Owen, \& Lewis, 2007) or during game-like situations involving activities such as catching a ball (Cowling \& Steele, 2001). In addition, direct comparison of the results is not possible due to differences in task demands. To our knowledge, no study has examined gender differences in knee kinematics and GRF when performing sport specific landing tasks during both unopposed and opposed conditions. The purpose of the present study was to examine the effect of opposition and gender on knee kinematics and GRF during landing from a volleyball block jump in male and female university volleyball players. 


\section{Method}

Participants

The participants were 6 female (Mean age $21.2 \pm 1.3$ years, mass $57.6 \pm 7.5 \mathrm{~kg}$ and height $164.8 \pm 7.5 \mathrm{~cm}$ ) and 6 male (Mean age $21.6 \pm 3.3$ years, mass $70.1 \pm 3.1 \mathrm{~kg}$ and height $175.7 \pm 8.6 \mathrm{~cm}$ ) university volleyball players. All participants had no previous history of hip, knee or ankle injury and were right leg dominant. Ethical approval was granted for the study by the University Ethics Committee and written consent forms were signed by all participants prior to data collection. The present study is part of a larger investigation examining landing biomechanics, of which some data has been previously published (Hughes et al., 2007).

\section{Measurement System}

An AMTI force platform sampling at $600 \mathrm{~Hz}$ was used to measure the GRF of the right (dominant) leg during landing. A time synchronized 12 camera Vicon 512 system (Vicon, Oxford, England) sampling at $120 \mathrm{~Hz}$ was used to determine 3D coordinates of 16 retro-reflective markers (25 $\mathrm{mm}$ diameter). Markers were placed directly on the skin over anatomical landmarks in accordance with the Vicon system's lower body plug-in gait marker set. From the location of the markers placed on the body, combined with required anthropometric measurements of each participant entered into the system, the Vicon system calculated the 3D coordinates of hip, knee and ankle joint centers. In the plug-in gait system, the measurement of knee flexion angle and valgus/varus angle was determined as the Euler angle of the shank segment reference frame relative to the thigh segment reference plane rotated in the order 1) flexion/extension, 2) valgus/varus, 3) internal/external rotation. 

lower limb stretching and running/jogging on a treadmill at self determined speeds. When this was completed, participants practiced the jumping and landing tasks until comfortable with the procedure. To carry out the landing task, a rope was fixed horizontally $5 \mathrm{~cm}$ in front of the force platform to act as a volleyball net at a height of $2.43 \mathrm{~m}$ for male participants and $2.24 \mathrm{~m}$ for female participants (height of a standard volleyball net). Also, a volleyball was suspended from the ceiling and positioned with the bottom of the ball $5 \mathrm{~cm}$ above the net ( $2.48 \mathrm{~m}$ for males and $2.29 \mathrm{~m}$ for females) and with the centre of the ball $10 \mathrm{~cm}$ in front of the line of the net (the other side of the net to where the participant (blocker) was standing). This was considered to be a typical position from which a volleyball is spiked from during a game. Participants were required to perform two landing tasks: unopposed volleyball block jump and landing and opposed volleyball block jump and landing. 1) Unopposed: At the start of each trial, the participant stood with their right foot on the force plate. The participant was then instructed to jump up and pretend to block the suspended volleyball. On landing, the right foot landed on the force plate. To standardize the unopposed blocking task, it was ensured that participants' hands reached the height of the top of the suspended volleyball in each trial. 2) Opposed: At the start of each trial, the participant stood with their right foot on the force plate. The participant then timed his/her blocking action in order to try to block the ball as it was spiked. In all trials, the person spiking the volleyball was of a similar playing standard to the blocker. The ball was spiked from the same suspended position in order to eliminate variation in the position and velocity of the ball. On landing, the right foot landed on the force plate. Data were recorded for three successful trials for each landing task for each participant. Trials where the entire right foot alone did not land on the force plate were discarded. 
Data Analysis

The data were filtered using a Woltring Filter. Through a frequency content analysis of the 3D coordinate data, the filter setting was determined as a low-pass filter of cut-off frequency $10 \mathrm{~Hz}$ and stop-band frequency of $30 \mathrm{~Hz}$. The GRF and knee angle in the sagittal (flexion/extension) and frontal (valgus/varus) planes were determined between initial ground contact (IC) and, depending on which occurred later in the trial, either maximum knee flexion or maximum knee valgus/varus angle (MAX) in each trial. Angular displacement mean data (IC, MAX and range of motion (ROM)) were based on 36 trials for both males and females (6 participants $\times 3$ trials $\times 2$ legs). GRF data were normalized to body weight (in Newtons) and mean data were based on 18 trials for both males and females ( 6 participants $\times 3$ trials $\times$ $1 \mathrm{leg}$ ). All statistical analysis was performed using SPSS version 14.0 (SPSS Inc, Chicago, IL). Mixed between-within participants analysis of variance (SPANOVA) was carried out on the data to examine the effects of the level of opposition and the effects of gender on angular displacement in the sagittal and frontal planes and normalized GRF, where the alpha level was set at $\mathrm{p}<0.05$.

\section{Results}

For all variables, there was no significant interaction between the level of opposition (unopposed/opposed) and gender (females/males) $(p>.05)$. All Figures show variables plotted against normalized time and against absolute mean trial time between IC and MAX. For the unopposed trials, absolute mean trial time was $0.203 \mathrm{~s} \pm 0.068$ for males and $0.213 \mathrm{~s}$ \pm 0.061 for females. For the opposed trials, absolute mean trial time was $0.190 \mathrm{~s} \pm 0.040$ for males and $0.194 \mathrm{~s} \pm 0.057$ for females. As there was no significant effect for level of opposition (Wilks Lambada $=.95, F=3.18, p=.08$, partial eta squared $=.05$ ) or for gender 
$(F=1.16, p=.29$, partial eta squared $=.02)$ for contact time, a mean trial time of $0.200 \mathrm{~s}$ was used.

\section{Effects of Opposition}

In the sagittal plane, there was a significant effect for level of opposition for knee flexion at IC (Wilks Lambada $=.86, F=9.68, p=.003$, partial eta squared $=.14$ ) with greater knee flexion observed at IC during unopposed trials than opposed trials (Table 1 and Figure 1). There was a significant effect for level of opposition (Wilks Lambada $=.77, F=17.6, p=$ .001 , partial eta squared $=.23$ ) for sagittal plane knee angle at MAX, with greater knee flexion at MAX observed during unopposed than opposed conditions (Table 1). This resulted in a significant effect for level of opposition (Wilks Lambada $=.86, F=9.61, p=.003$, partial eta squared $=.14$ ) for ROM of knee angle in the sagittal plane, with greater ROM of knee flexion observed during unopposed than opposed conditions (Table 1).

\section{Table 1 about here.}

Figure 1 about here.

In the frontal plane, there was no significant effect for level of opposition (Wilks Lambada $=1.00, F=.001, p=.97$, partial eta squared $=.001)$ for the knee valgus angle at IC, no significant effect for level of opposition (Wilks Lambada $=.95, F=2.80, p=.10$, partial eta squared $=.05$ ) for MAX knee valgus angle and no significant effect for level of opposition (Wilks Lambada $=.94, F=4.05, p=.06$, partial eta squared $=.07$ ) for ROM of knee angle in the frontal plane (Table 1 and Figure 2). 
Figure 2 about here.

For most of the landing period, the normalized GRF was greater for opposed trials than unopposed trials (Figure 3). There was no significant effect for level of opposition (Wilks Lambada $=.93, F=2.17, p=.15$, partial eta squared $=.07$ ) for normalized GRF at MAX. For peak normalized GRF, there was a significant effect for level of opposition (Wilks observed during opposed conditions than unopposed conditions (Table 2).

Table 2 about here.

Effects of Gender

In the sagittal plane, there was no significant effect for gender $(F=3.65, p=.06$, partial eta squared $=.06)$ for knee flexion at IC. There was a significant effect for gender $(F=$ $13.3, p=.01$, partial eta squared $=.19$ ) for sagittal plane knee angle at MAX, with females

In the frontal plane, females tended to contact the ground with the knee in a valgus 
between IC and MAX position. In contrast, males tended to contact the ground with the knee in a valgus position and moved into a varus position (positive values for knee angle in the frontal plane) at MAX (Table 1 and Figure 2). There was no significant effect for gender $(F=$ $.35, p=.56$, partial eta squared $=.01$ ) for the knee valgus angle at IC. For MAX knee valgus angle, there was a significant effect for gender $(F=32.3, p=.001$, partial eta squared $=.36)$ with females exhibiting a greater MAX knee valgus angle than males (Table 1). This resulted in a significant effect for gender $(F=38.6, p=.001$, partial eta squared $=.40)$ for ROM of knee angle in the frontal plane, with females displaying a greater ROM of knee valgus angle than males (Table 1).

With regard to normalized GRF (Figure 3), the overall shapes of the curves were similar for males and females, where an increase was shown during approximately the first $40 \%$ of the landing phase followed by decrease during approximately the final $60 \%$ of landing. For most of the landing period, the normalized GRF was greater for males than females. However, there was no significant effect for gender $(F=.07, p=0.79$, partial eta squared $=.02)$ for normalized GRF at MAX and no significant effect for gender $(F=1.43, p$ $=.24$, partial eta squared $=.05$ ) for peak normalized GRF (Table 2).

\section{Discussion}

\section{Effects of Opposition}

The results indicate significant differences in sagittal plane kinematics between unopposed and opposed trials. There was a significant effect for level of opposition in knee flexion at IC, with greater knee flexion at IC exhibited during unopposed conditions than opposed conditions. In addition, the effect for opposition was greater for females than males where females exhibited on average a $4.4^{\circ}$ reduction in knee flexion at IC when opposition 
was included in the task compared to a $0.9^{\circ}$ reduction in males. ACL strain is likely to be increased with reduced knee flexion (Li et al., 1999; Nunley et al., 2003), therefore during unopposed trials participants may be more able to increase knee flexion at IC compared to opposed trials to reduce the likelihood of ACL strain. This may be due to participants having greater visual awareness of when ground contact is likely to take place during unopposed trials. Since participants did not need to spend as much time and attention watching the ball being spiked during unopposed trials, participants could anticipate ground contact more easily and therefore prepare for a safer landing through flexing the knee slightly before IC. There was a significant effect for level of opposition for MAX knee flexion and ROM of knee flexion, with greater knee flexion exhibited during unopposed conditions than opposed conditions. The results of the present study indicate values of maximum knee flexion measured during unopposed trials were nearer to values reported by previous studies where participants performed unopposed landing than those measured during opposed conditions. For example, mean maximum knee flexion of $88.9^{\circ} \pm 11.4$ for males and $78.3^{\circ} \pm 13.4$ for females were reported by Kernozek et al. (2005) compared to $67.2^{\circ} \pm 12.9$ for males and $78.0^{\circ} \pm 8.1$ for females during unopposed trials and $62.1^{\circ} \pm 11.6$ for males and $68.2^{\circ} \pm 12.2$ for females during opposed trials. The greater knee flexion exhibited during unopposed conditions compared to opposed conditions may be due to participants consciously increasing their knee flexion during unopposed trials in an attempt to reduce the impact of the GRF during landing and therefore reduce the risk of injury. However, during opposed trials, due to the greater attentional demand of effectively performing the blocking action, participants were, perhaps, less able to consciously increase the amount of knee flexion during landing. These results indicate that sagittal plane kinematics changed significantly with the introduction of opposition to the landing task and highlight the need for ecologically valid 
task demands in studies designed to examine differences in the incidence of injuries between males and females in specific sports.

The results indicate no significant effect for level of opposition in knee valgus angle during landing. These results indicate that differences in frontal plane kinematics between males and females during landing were consistent between unopposed and opposed conditions. The values of maximum knee valgus angle reported in this study are different to previous results but as with the sagittal plane kinematics, the results of the present study indicate values of maximum knee valgus angle measured during unopposed trials were nearer to values reported by previous studies where participants performed unopposed landing than those measured during opposed conditions. For example, Ford et al. (2004) reported maximum knee valgus (-ve) / varus (+ve) angle values of $-14.3^{\circ} \pm 2.0$ for males and $-20.1^{\circ} \pm$ 2.5 for females, compared to $-2.2^{\circ} \pm 5.3$ for males and $-13.9^{\circ} \pm 11.3$ for females during unopposed trials and $-2.9^{\circ} \pm 7.9$ for males and $-10.4^{\circ} \pm 7.7$ for females during opposed trials in this study. There are a number of possible reasons for these differences which include participants' age and playing standard and the method of measuring the knee valgus angle. In Ford et al. (2004) the participants used were high school athletes whereas university athletes were used in this study. The valgus angle measured in Ford et al. (2004) was determined from markers placed on the skin over the greater trochanter, lateral epicondyle of the knee and the lateral malleolus of the ankle, whereas in this study, the valgus angle was based on estimated hip, knee and ankle joint centers using the Vicon plug-in gait model.

There was a significant effect for level of opposition in peak normalized GRF with greater normalized GRF exhibited during opposed conditions compared to unopposed conditions. When performing a landing from a jump, a participant is required to effectively reduce both their angular and linear momentum to zero. Having been stuck by the ball while in flight during the opposed trials, participants are likely to have a greater angular momentum 
about their centre of gravity when they make contact with the ground during opposed trials than during unopposed trials. This means that participants must reduce a larger angular momentum, as well as their linear momentum, to zero during opposed trials. This greater momentum of the body at IC may contribute to greater GRF during opposed trials. Also, as stated previously, the reduced GRF during unopposed trials compared to opposed trials may be due to the greater ability of participants to consciously increase knee flexion during unopposed trials as a result of the reduced attentional demand of the task. This increased knee flexion may result in a reduction in the GRF acting on the body during landing and therefore reduce the likelihood of injury from high GRF.

Effects of Gender

There was no significant effect for gender for knee flexion at IC, contrary to a number of previous studies (Decker et al., 2003; James et al., 2004; Yu et al., 2006). The values recorded in this study for knee flexion at IC are also slightly less than those reported in previous research. For example, Decker et al. (2003) reported knee flexion angles at IC of $30.0 \pm 7.7^{\circ}$ in males and $22.8 \pm 8.0^{\circ}$ in females, compared to $20.3 \pm 4.7^{\circ}$ for males and $19.5 \pm$ $6.9^{\circ}$ for females in the present study during unopposed trials. The reasons for this difference with the previous literature may be due to differences in the measuring systems and participants used since this study used experienced volleyball players whereas Decker et al. (2003) examined recreational athletes. Also, during unopposed trials there was a relatively small difference between males and females for knee flexion at IC (males $0.8^{\circ}$ greater than females) whereas during opposed trials there was a larger gender difference (males $4.3^{\circ}$ greater than females). There was a significant effect for gender for MAX knee flexion and ROM of knee flexion, with greater knee flexion exhibited by females compared to males. Some previous studies have also found that females displayed greater knee flexion than males 
during landing (Decker et al., 2003) whereas other found reduced knee flexion in females compared to males (Salci et al., 2004; Yu et al., 2006). In the present study, the greater knee flexion exhibited by females compared to males may be associated with the greater knee valgus shown by females than males, whereby females are less able to resist angular displacement on the knee during landing and therefore display reduced dynamic stability of the knee joint, which may be associated with ACL injury.

The results indicate significant differences in frontal plane kinematics between males and females. There was no significant effect for gender in knee valgus at IC, which is similar to the findings previous research (Kernozek et al., 2005). However, there was a significant effect for gender for MAX knee valgus and ROM of knee valgus, with females displaying greater knee valgus angle than males during landing. Greater knee valgus angle in females has also been found by a number of other studies examining frontal plane knee kinematics during unopposed landing tasks (Ford et al., 2003; Kernozek et al., 2005). Greater knee valgus angle during landing may indicate increased risk of ACL injury in females compared to males.

For most of the landing period, the normalized GRF was greater for males than females. This is contrary to a number of previous studies examining gender differences in normalized GRF during landing (Kernozek et al., 2005; Salci et al., 2004; Yu et al., 2006). The difference in the findings of the present study and previous studies is likely to be due to differences in task demands participants were required to perform. Typically, previous studies have examined drop-jump landings from the same set height for males and females whereas the present study examined a sport specific volleyball block jump landing, where males and females were more likely to land from a jump height typical of what they are likely to perform during their sport. 
In conclusion, differences in sagittal plane knee kinematics and GRF during opposed 360 and unopposed trials suggest that coaches should implement training programs that involve 361 ecologically valid landing maneuvers. Future research into landing kinematics and kinetics 362 should include opposition during the landing task as the effect of opposition may 363 significantly alter participants' neuromuscular responses during landing, particularly in the sagittal plane. Differences in frontal plane kinematics between males and females however, appear to be consistent in unopposed and opposed conditions. Therefore the results of this study may validate the results of many other studies (Ford et al., 2003; Kernozek et al., 2005; Malinzak et al., 2001) which have investigated gender differences in frontal plane knee kinematics during landing in unopposed conditions. 


\section{References}

Arendt, E. A., \& Dick, R. (1995). Knee injury patterns among men and women in collegiate basketball and soccer. The American Journal of Sports Medicine, 23, 694-701.

Boden, B. P., Dean, G. S., Feagin, J. A., \& Garett, W. E. (2000). Mechanisms of anterior cruciate ligament injury. Orthopedics, 23, 573-578.

Chandy, T. A., \& Grana, W. A. (1985). Secondary school athletic injury in boys and girls: a three-year comparison. Physician and Sports Medicine, 13, 314-316.

Chappell, D. J., Yu, B., Kirkendall, D. T., \& Garrett, W. E. (2002). A comparison of knee kinetics between male and female recreational athletes in stop-jump tasks. The American Journal of Sports Medicine, 30(2), 261-267.

Chen, H. C., Schultz, A. B., Ashton-Miller, J. A., Giordani, B., Alexander, N. B., \& Guire, K. E. (1996). Stepping over obstacles: Dividing attention impairs performance of old more than young adults. Journals of Gerontology Series A - Biological Sciences and Medical Sciences, 51(3), 116-122.

Cowling, E. J., \& Steele, J. R. (2001). Is lower limb muscle synchrony during landing affected by gender? Implications for variations in ACL injury rates. Journal of Electromyography and Kinesiology, 11, 263-268.

Davila, M. G., Garcia, P. L., Montilla, J. P., \& Ruiz, F. J. R. (2006). Effect of opposition on the handball jump shot. Human Movement Studies, 51(4), 257-275. 
Decker, M. J., Torry, M. R., Wyland, D. J., Sterett, W. I., \& Steadman, J. R. (2003). Gender differences in lower extremity kinematics, kinetics and energy absorption during landing. Clinical Biomechanics, 18, 662-669.

Devita, P., \& Skelly, W. A. (1992). Effect of landing stiffness on joint kinetics and energetics in the lower extremity. Medicine and Science in Sport and Exercise, 24, 108-115.

Faegin, J. A. (1988). Isolated anterior cruciate injury. In J. A. Faegin (Ed.), The Crucial Ligaments (pp. 15-23). New York: Churchill Livingstone.

Ferretti, A., Papandrea, P., Conteduca, F., \& Mariani, P. P. (1992). Knee ligament injuries in volleyball players. The American Journal of Sports Medicine, 20, 203-207.

Ford, K. R., Myer, G. D., \& Hewett, T. E. (2003). Valgus knee motion during landing in high school female and male basketball players. Medicine and Science in Sport and Exercise, 35, 1745-1750.

Gray, J., Taunton, J. E., McEnzie, D. C., Clement, D. B., McConkey, J. P., \& Davidson, R. G. (1985). A survey of injuries to the anterior cruciate ligament of the knee in female basketball players. International Journal of Sports Medicine, 6, 314-316.

Griffin, L. Y., Angel, J., Albohm, M. J., Arendt, E. A., Dick, R. W., Garrett, W. E., et al. (2000). Noncontact anterior cruciate ligament injuries: risk factors and prevention strategy. Journal of the American Academy of Orthopaedic Surgeons, 8(3), 141-150.

Gwinn, D. E., Wilckens, J. H., McDevitt, E. R., Ross, G., \& Kao, T. C. (2000). The relative incidence of anterior cruciate ligament injury in men and women at the United States naval academy. The American Journal of Sports Medicine, 28, 98-102. 
Hewett, T. E., Stroupe, A. L., Nance, T. A., \& Noyes, F. R. (1996). Plyometric training in female athletes: decreased impact forces and increasing hamstring torques. The American Journal of Sports Medicine, 24(6), 765-773.

Hopper, D., \& Elliot, B. (1993). Lower limb and back injury patterns of elite netball players. Sports Medicine, 16, 148-162.

Hughes, G., Watkins, J., Owen, N., \& Lewis, M. (2007). Gender differences in knee kinematics during landing from volleyball block jumps. Human Movement Studies, $53(1), 1-20$.

James, C. R., Sizer, P. S., Starch, D. W., Lockhart, T. E., \& Slauterbeck, J. (2004). Gender differences among sagittal plane knee kinematics and ground reaction force characteristics during a rapid sprint and cut manoeuvre. Research Quarterly for Exercise and Sport, 8, 31-39.

Johnson, R. J. (1988). Prevention of anterior cruciate ligament injuries. In J. A. Faegin (Ed.), The Critical Ligaments (pp. 349-356). New York: Churchill Livingston.

Kernozek, T. W., Torry, M. R., Van Hoof, H., Cowley, H., \& Tanner, S. (2005). Gender differences in frontal plane and sagittal plane biomechanics during drop landings. Medicine and Science in Sport and Exercise, 37(6), 1003-1012.

Lajoie, Y., Teasdale, N., Bard, C., \& Fleury, M. (1993). Attentional demands for static and dynamic equilibrium. Experimental Brain Research, 97(1), 139-144. 
Li, G., Rudy, T. W., Sakane, M., Kanamori, A., Ma, C. B., \& Woo, S. L. Y. (1999). The importance of quadriceps and hamstring muscle loading on knee kinematics and insitu forces in the ACL. Journal of Biomechanics, 32, 395-400.

Lidenfeld, T. N., Schmitt, D. J., Hendy, M. P., Mangine, R. E., \& Noyes, F. R. (1994). Incidence of injury in indoor soccer. The American Journal of Sports Medicine, 22, $354-371$.

Malinzak, R. A., Colby, S. M., Kirkendall, D. T., Yu, B., \& Garrett, W. E. (2001). A comparison of knee joint motion patterns between men and women in selected athletic tasks. Clinical Biomechanics, 16, 438-445.

Malone, T. R., Hardaker, W. T., Garrett, W. E., Feagin, J. A., \& Bassett, F. H. (1993). Relationship of gender to anterior cruciate ligament injuries in intercollegiate basketball players. Journal of the Southern Orthopaedic Association, 2, 36-39.

McNair, P., Marshall, R., \& Matheston, J. (1993). Important features associated with acute anterior cruciate injury. The New Zealand Medical Journal, 103, 537-539.

Miller, M. D. M., Cooper, D. E., \& Warner, J. J. P. (1995). Review of Sports Medicine and Arthroscopy. Philadelphia, PA: W.B. Saunders.

Mykelbust, G., Maehlum, S., Engbretsen, L., Strand, T., \& Solheim, E. (1997). Registration of cruciate ligament injuries in Norwegian top level team handball: a prospective study covering two seasons. Scandinavian Journal of Medicine and Science in Sports, 7, 289-292. 
Nunley, R. M., Wright, D., Renner, J. B., Yu, B., \& Garrett, W. E. (2003). Gender comparison of patella tendon tibial shaft angle with weight bearing. Research in Sports Medicine: An International Journal, 11(3), 173-185.

Olsen, O. E., Mykelbust, G., Engebretsen, L., \& Bahr, R. (2004). Injury mechanisms for anterior cruciate ligament injuries in team handball: A systematic video analysis. The American Journal of Sports Medicine, 32(4), 1002-1012.

Salci, Y., Kentel, B. B., Heycan, C., Akin, S., \& Korkusus, F. (2004). Comparison of landing manoeuvres between male and female college volleyball players. Clinical Biomechanics, 19(6), 622-628.

Smith, B. A., Livesay, G. A., \& Woo, S. L. Y. (1988). Biology and biomechanics of the anterior cruciate ligament. Clinical Sports Medicine, 12, 637-666.

Swartz, E. E., Decoster, L. C., Russell, P. J., \& Croce, R. V. (2005). Effects of development stage and sex on lower extremity kinematics and vertical ground reaction forces during landing. Journal of Athletic Training, 40(1), 9-14.

Yu, B., Lin, C. F., \& Garrett, W. E. (2006). Lower extremity biomechanics during the landing of a stop-jump task. Clinical Biomechanics, 21, 297-305.

Yu, B., McClure, S. B., Onate, J. A., Guskiewicz, K. M., Kirkendall, D. T., \& Garrett, W. E. (2005). Age and gender effects on lower extremity kinematics of youth soccer players in a stop-jump task. The American Journal of Sports Medicine, 33(9), 13561364. 
There is no financial interest in the research.

Corresponding Author: Dr. Gerwyn Hughes.

Postal Address: $\quad$ Division of Sport, Health and Exercise, University of Hertfordshire,

477 Email:

g.hughes@herts.ac.uk

Telephone:

$+441707289430$

479

480 Author 2:

Prof. James Watkins.

Department of Sports Science, Swansea University, Singleton Park, Swansea, SA2 8PP.

483

Email:

j.watkins@swansea.ac.uk

484

485

Author 3:

Mr. Nick Owen.

486

Postal Address:

Department of Sports Science, Swansea University, Singleton Park,

487 Swansea, SA2 8PP.

488

Email:

n.owen@swansea.ac.uk 
Table 1. Group mean results for knee flexion/extension and valgus/varus (- valgus; + varus) angles at IC, MAX and ROM for males and females during unopposed and opposed trials (Mean \pm standard deviation).

\begin{tabular}{|c|c|c|c|c|c|}
\hline & \multicolumn{3}{|c|}{ Males } & \multicolumn{2}{|c|}{ Females } \\
\hline & & Unopposed $\left({ }^{\circ}\right)$ & Opposed $\left(^{\circ}\right)$ & Unopposed $\left({ }^{\circ}\right)$ & Opposed $\left({ }^{\circ}\right)$ \\
\hline \multirow{3}{*}{ Flexion } & $\mathrm{IC} *$ & $20.3 \pm 4.7$ & $19.4 \pm 6.4$ & $19.5 \pm 6.9$ & $15.1 \pm 6.2$ \\
\hline & MAX $* \dagger$ & $67.2 \pm 12.9$ & $62.1 \pm 11.6$ & $78.0 \pm 8.1$ & $68.2 \pm 12.2$ \\
\hline & $\mathrm{ROM} * \dagger$ & $46.9 \pm 14.9$ & $42.7 \pm 13.9$ & $58.6 \pm 7.4$ & $53.1 \pm 13.1$ \\
\hline \multirow{4}{*}{$\mathrm{Val} / \mathrm{var}$} & IC & $-2.2 \pm 5.3$ & $-2.8 \pm 5.9$ & $-2.1 \pm 3.4$ & $-1.6 \pm 2.8$ \\
\hline & $\operatorname{MAX}_{\mathrm{VAL}^{\dagger}}^{\dagger}$ & $-2.2 \pm 5.3$ & $-2.9 \pm 7.9$ & $-13.9 \pm 11.3$ & $-10.4 \pm 7.7$ \\
\hline & MAXVAR $_{\text {VAR }}$ & $1.0 \pm 9.6$ & $0.6 \pm 9.1$ & N/A & N/A \\
\hline & $\mathrm{ROM}^{\dagger}$ & $3.2 \pm 8.0$ & $3.5 \pm 9.6$ & $11.8 \pm 10.3$ & $8.8 \pm 7.8$ \\
\hline
\end{tabular}

496

$497 *$ : Significant effect between unopposed and opposed trials $(\mathrm{p}<0.05)$.

$498 \dagger$ : Significant effect between males and females $(p<0.05)$.

499 
500 Table 2. Group mean results for normalized GRF at MAX and peak (Mean \pm standard 501 deviation).

\section{MAX GRF (BW) Peak GRF (BW)}

\begin{tabular}{cccc}
\hline \multirow{2}{*}{ Males } & Unopposed & $0.752 \pm 0.194$ & $1.561 \pm 0.663^{*}$ \\
& Opposed & $0.972 \pm 0.415$ & $1.861 \pm 0.595^{*}$ \\
\hline \multirow{2}{*}{ Females } & Unopposed & $0.873 \pm 0.210$ & $1.457 \pm 0.477^{*}$ \\
& Opposed & $0.894 \pm 0.378$ & $1.631 \pm 0.427^{*}$ \\
\hline
\end{tabular}

502

503

*: Significant effect between unopposed and opposed trials.

504 
505 Figure Captions

506

507 Figure 1. Knee flexion $\left(\theta_{\mathrm{f}}\right)$ between IC and MAX for males and females during unopposed 508 and opposed trials.

509 Figure 2. Knee valgus/varus $\left(\theta_{\mathrm{v}}\right)$ between IC and MAX for males and females during 510 unopposed and opposed trials.

511 Figure 3. Normalized GRF between IC and MAX for males and females during unopposed 512 and opposed trials.

513 